\title{
VISCOSITÉ DE LA MATIËRE GRASSE DU BEURRE
}

\author{
par
}

\section{GORIAEW}

Chargé de la chaire "la Biochimie du lait» à l'Institut des Ingénieurs d'Industrie laitière à Leningrad (U. R. S. S.).

L'emploi de l'écrémeuse dans la fabrication du beurre fondu pour obtenir la séparation rapide de la matière grasse du beurre en toute sa pureté, dégagée de l'eau et de la partie albumineuse du beurre, au lieu du long procédé d'autrefois (12 à 18 heures) qui consistait à laisser s'accomplir spontanément la montée du beurre dans la cuve, nécessite l'appréciation de la viscosité de la matière grasse du beurre.

En fait de renseignements à ce sujet, nous trouvons chez Pleissner [1] les indications suivantes :

La détermination de la viscosité de la matière grasse du beurre est effectuée d'après la formule suivante : $\rho=\frac{\mathrm{s.t}}{\tau}$, dans laquelle p - représente la viscosité, s - le poids spécifique de la graisse, $\tau-$ le temps d'écoulement de la graisse à $20^{\circ}$. Les données de l'auteur sont réunies dans le tableau ci-dessous (tableau I) :

TABLEAU I.

\begin{tabular}{|c|c|c|c|}
\hline Température & $\mathrm{t}$ & s & p \\
\hline $25^{\circ} 3$ & 263 & 0,9186 & 43,9 \\
\hline $29^{\circ} 8$ & 223 & 0,9155 & 37,1 \\
\hline $32^{\circ} 9$ & 186 & 0,9134 & 30,9 \\
\hline $40^{\circ} 2 \ldots$ & 157 & 0,9684 & 25,9 \\
\hline $100^{\circ} 0 \ldots \ldots \ldots \ldots \ldots \ldots \ldots \ldots$ & 30 & 0,8664 & 4,7 \\
\hline
\end{tabular}

La viscosité de la matière grasse du beurre et celle de l'oléomargarine ont été très soigneusement déterminées, par WHITE et Twining [2]. Ils se servirent à ce propos du viscosimètre à pression alternative, tout en mettant en évidence les erreurs en énergie cinétique.

En comparaison des autres recherches de ce genre, les leurs seraient peut-être à noter comme très précises. Ainsi la viscosité de la matière grasse du beurre à $40^{\circ} \mathrm{C}$. est de 0,320 poises. Les mêmes auteurs trouvent la fluidité de la matière grasse du beurre d'après la fonction de la température. 
Nous présentons nos mesures pour pouvoir exprimer graphiquement cette même dépendance dans les limites de $30^{\circ}$ à $100^{\circ} \mathrm{C}$.

Les mesures de la viscosité furent exécutées à l'aide du viscosimètre Ostwald, celui-ci, ayant été soumis d'avance à l'épreuve $R$. Le temps de l'écoulement de l'eau marquait la constante 6 secondes. Pour l'élimination de l'eau des échantillons de la matière grasse l'on eut recours à la méthode habituelle $\mathrm{C}$.

Le poids spécifique de la matière grasse du beurre a été déterminé sur la balance Mohr et Westphal et sa viscosité calculée en valeurs absolues d'après la formule :

dans laquelle :

$$
\eta=\frac{d \cdot t}{d_{w} \cdot t_{w}} \cdot \eta_{w}
$$

$\eta_{i}$ indique la viscosité de la matière grasse.

t indique le temps de l'écoulement de la matière grasse.

d indique son poids spécifique.

$\mathrm{d}_{\mathrm{w}}$ indique le poids spécifique de l'eau à $20^{\circ} \mathrm{C} .=0,9982$.

$t_{\mathrm{w}}$ indique le temps indispensable à l'écoulement de l'eau.

$\eta_{\mathrm{w}}$ indique la viscosité de l'eau à $20^{\circ} \mathrm{C}$. $=1,005$ centipoises.

Les quatre échantillons différents de beurre nous ont fourni les données réunies dans le tableau II.

TABLEAU II.

\begin{tabular}{|c|c|c|c|c|c|c|c|c|c|}
\hline \multirow{2}{*}{$\begin{array}{l}\text { Echan- } \\
\text { tillons } \\
\mathrm{N}^{\circ}\end{array}$} & & \multicolumn{8}{|c|}{ Température } \\
\hline & & $30^{\circ}$ & $40^{\circ}$ & $50^{\circ}$ & $60^{\circ}$ & $70^{\circ}$ & $80^{\circ}$ & $90^{\circ}$ & $100^{\circ}$ \\
\hline$I \ldots$ & $\begin{array}{l}d \\
t \\
\eta\end{array}$ & $\begin{array}{c}0,907 \\
168,4 \\
25,62\end{array}$ & $\begin{array}{c}0,900 \\
115,3 \\
17,41\end{array}$ & $\begin{array}{l}0,895 \\
83,3 \\
12,51\end{array}$ & $\begin{array}{c}0,890 \\
60,7 \\
9,06\end{array}$ & $\begin{array}{c}0,885 \\
47,3 \\
7,02\end{array}$ & $\begin{array}{c}0,880 \\
40,3 \\
5,95\end{array}$ & $\begin{array}{c}0,875 \\
35,3 \\
5,42\end{array}$ & $\begin{array}{c}0,866 \\
30,0 \\
4,36\end{array}$ \\
\hline II .. & $\begin{array}{l}d \\
t \\
\eta\end{array}$ & $\begin{array}{c}0,915 \\
168,6 \\
25,89\end{array}$ & $\begin{array}{c}0,906 \\
114,6 \\
17,42\end{array}$ & $\begin{array}{c}0,900 \\
80,8 \\
12,20\end{array}$ & $\begin{array}{c}0,895 \\
62,3 \\
9,36\end{array}$ & $\begin{array}{c}0,883 \\
45,7 \\
6,77\end{array}$ & $\begin{array}{c}0,881 \\
38,4 \\
5,68\end{array}$ & $\begin{array}{c}0,875 \\
31,3 \\
4,60\end{array}$ & $\begin{array}{c}0,866 \\
27,4 \\
3,98\end{array}$ \\
\hline III . . & $\begin{array}{l}d \\
t \\
\eta\end{array}$ & - & $\begin{array}{c}0,905 \\
113,3 \\
17,21\end{array}$ & $\begin{array}{c}\quad 0,897 \\
83,6 \\
12,59\end{array}$ & $\begin{array}{c}0,890 \\
61,0 \\
9,41\end{array}$ & $\begin{array}{c}0,882 \\
50,3 \\
7,44\end{array}$ & $\begin{array}{c}0,874 \\
41,6 \\
6,10\end{array}$ & $\begin{array}{c}0,866 \\
31,5 \\
4,58\end{array}$ & $\begin{array}{c}0,863 \\
29,6 \\
4,29\end{array}$ \\
\hline IV & $\begin{array}{l}d \\
t \\
\eta\end{array}$ & $\begin{array}{c}0,912 \\
168,9 \\
25,85\end{array}$ & $\begin{array}{c}0,904 \\
114,8 \\
17,36\end{array}$ & $\begin{array}{c}0,898 \\
82,4 \\
12,42\end{array}$ & $\begin{array}{c}0,892 \\
61,4 \\
9,19\end{array}$ & $\begin{array}{c}0,884 \\
47,7 \\
7,08\end{array}$ & $\begin{array}{c}0,878 \\
40,4 \\
5,95\end{array}$ & $\begin{array}{c}0,872 \\
32,8 \\
4,80\end{array}$ & $\begin{array}{c}0,866 \\
28,5 \\
4,14\end{array}$ \\
\hline $\begin{array}{l}\text { En } \\
\text { moy. }\end{array}$ & $\eta$ & 25,78 & 17,35 & $12,43 \mathrm{~s}$ & 9,26 & 7,08 & 5,92 & 4,87 & 4,19 \\
\hline
\end{tabular}


Nous basant là-dessous, nous sommes parvenus à calculer la viscosité cinématique $\eta / d$ et la fluidité $1 / \eta$.

TABLEAU III

\begin{tabular}{|c|c|c|c|c|c|c|c|c|}
\hline $\begin{array}{l}\text { Température } \\
\text { Degrés C. }\end{array}$ & $30^{\circ}$ & $40^{\circ}$ & $50^{\circ}$ & $60^{\circ}$ & $70^{\circ}$ & $-80^{\circ}$ & $90^{\circ}$ & $100^{\circ}$ \\
\hline $\begin{array}{l}\text { Viscosité }(\eta) \\
\text { (poises) }\end{array}$ & 0,258 & 0,173 & 0,124 & 0,093 & 0,071 & 0,059 & 0,049 & 0,042 \\
\hline $\begin{array}{l}\text { Viscosité ci- } \\
\text { nématique } \eta / d\end{array}$ & 0,283 & 0,192 & 0,138 & 0,104 & 0,080 & 0,68 & 0,055 & 0,048 \\
\hline Fluidité $1 / \eta$ & 3,9 & 5,7 & 8,0 & 10,8 & 14,1 & 16,9 & 20,5 & 23,8 \\
\hline
\end{tabular}

Résumé

1. La détermination de la viscosité de la matière grasse du beurre a été faite dans des intervalles de température de $30^{\circ}$ à $100^{\circ} \mathrm{C}$.

2. La viscosité cinématique et la fluidité de la matière grasse du beurre ont été calculées. La fluidité de la matière grasse du beurre dans l'intervalle de $50^{\circ}$ à $100^{\circ}$ démontre la fonction linéaire de la température.

3. A $30^{\circ}$, les déterminations de la viscosité deviennent plus compliquées à cause d'une solidification lente et partielle des glycérides; c'est pourquoi nous nous vîmes privés de la possibilité d'accomplir des déterminations de la viscosité quelque peu sûres, pour des températures de la matière grasse inférieures à $30^{\circ} \mathrm{C}$.

\section{BIBLIOGRAPHIE.}

[1] Pleissner. Arch. d. Pharm., 242, 1904, 24.

[2] White et Twining. Journal Industrial and Engineering Chemistry, b. $\mathrm{n}^{\circ} 5 ; 1913,5568$.

(Traduit du russe par O. KaRAKONOWSKY.) 